\title{
Editorial Statistics and Best Reviewer Awards 2020 for the Journal of Korean Neurosurgical Society
}

\author{
Moonyoung Chung, ${ }^{1}$ Chul-Kee Park, ${ }^{2}$ Hee-Jin Yang ${ }^{3}$ \\ Managing Editor,' Journal of Korean Neurosurgical Society; Department of Neurosurgery, Soonchunhyang University Bucheon Hospital, Soon- \\ chunhayng University, Bucheon, Korea \\ Associate Editor, ${ }^{2}$ Journal of Korean Neurosurgical Society; Department of Neurosurgery, Seoul National University Hospital, Seoul, Korea \\ Editor in Chief, Journal of Korean Neurosurgical Society; Department of Neurosurgery, Seoul National University Boramae Hospital, Seoul, \\ Korea
}

\section{EDITORIAL STATISTICS}

In the last year, more than 350 articles were submitted to the Journal of Korean Neurosurgical Society (JKNS). The editorial office of the JKNS investigated the records regarding who had participated as reviewers for the JKNS in 2020. From January 1 to November 12, a total of 582 requests to review articles were sent to 193 reviewers. Of these, 446 review requests were completed, corresponding to $76.6 \%$ of the total requests. The average duration of the first review was $10.5 \pm 4.8$ days (Fig. 1). The reviewers were divided into two groups-those who completed the review request in 1-10 days were categorized as "fast reviewers," while those who completed the review request after 10 days were categorized as "slow reviewers." Group analyses revealed a trend in which the fast reviewers were requested to review more articles (3.2 articles/reviewer) than slow reviewers (2.7 articles/reviewer, independent t-test, $P=0.052)$. However, there was no statistical difference between the two groups regarding the numbers of review requests completed (2.5 articles in fast reviewers and 2.1 articles in slow reviewers, independent t-test, $P=0.126)$.

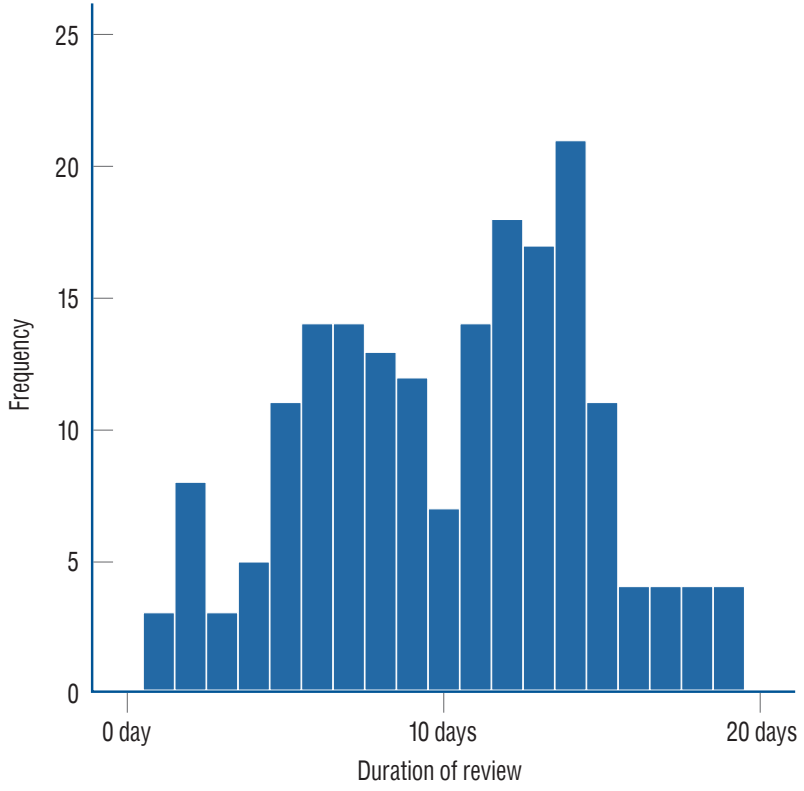

Fig. 1. Distribution of the time taked for the completion of the first review. This figure shows the distribution of the time taken (days) for the completion of the first reviews in the Journal of Korean Neurosurgical Society. The mean time for the first review was 10.5 days. Two groups can be distinguished in the figure : no more than 10 days and more than 10 days for completion of the review process.

- Received : December 29, 2020 •Accepted : December 29, 2020

- Address for reprints : Hee-Jin Yang

Department of Neurosurgery, Seoul National University Boramae Hospital, 20 Boramae-ro 5-gil, Dongjak-gu, Seoul 07061, Korea Tel : +82-2-870-2303, Fax : +82-2-870-3863, E-mail : nsyangdr@gmail.com, ORCID : http://orcid.org/0000-0002-6413-1363

This is an Open Access article distributed under the terms of the Creative Commons Attribution Non-Commercial License (http://creativecommons.org/licenses/by-nc/4.0) which permits unrestricted non-commercial use, distribution, and reproduction in any medium, provided the original work is properly cited. 


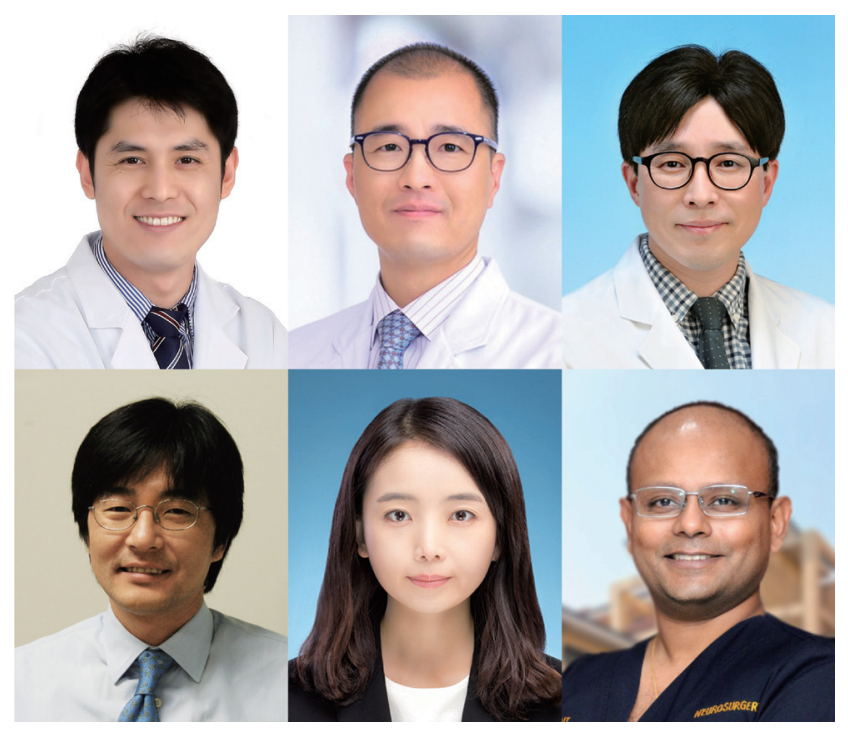

Fig. 2. The best reviewer in 2020. The best reviewers from top left to bottom right : Man Kyu Choi, Chi Heon Kim, Euihyun Kim, Seung Ki Kim, Hye Ran Park, and Kamalanathan Palaniandy.

Among the reviewers, those who reviewed a particularly large number of papers and faithfully commented on their requests were selected as the JKNS best reviewers in 2020 consistent with previously years ${ }^{1)}$. We have introduced the reviewers below with our honor. The list is arranged in alphabetical according to the reviewers' last names (Fig. 2).

\section{Man Kyu Choi \\ Kyung Hee University College of Medicine Spine}

\section{Euihyun Kim}

Yonsei University College of Medicine

Tumor

\section{Kamalanathan Palaniandy Hye Ran Park}

Universiti Kebangsaan, Malaysia

Neurotrauma

\section{Chi Heon Kim}

Seoul National University Hospital Spine

\section{Seung Ki Kim}

Seoul National University Hospital Pediatric

Soonchunhyang University Seoul Hospital Stereotactic and functional

\section{CONFLICTS OF INTEREST}

No potential conflict of interest relevant to this article was reported.

\section{AUTHOR CONTRIBUTIONS}

\author{
Conceptualization : MC, CKP, HJY \\ Data curation : MC \\ Formal analysis : $\mathrm{MC}$ \\ Funding acquisition : $\mathrm{MC}$ \\ Methodology : MC, CKP, HJY \\ Project administration : HJY \\ Visualization : MC, HJY \\ Writing - original draft : $\mathrm{MC}, \mathrm{HJY}$ \\ Writing - review \& editing : MC, CKP, HJY
}

\section{ORCID}

\section{IMPORTANCE OF GOOD REVIEWERS FOR THE PROGRESS OF THE JKNS}

The JKNS, a journal with 49 -years history since 1972 , is being classified as an international journal thanks to the efforts of all of the members of the Korean Neurosurgical Society. The continuous increase in the impact factor of the JKNS speaks for itself. The Editor-in-Chief's passion is an important
Moonyoung Chung https://orcid.org/0000-0003-4733-6321 Chul-Kee Park https://orcid.org/0000-0002-2350-9876

Hee-Jin Yang https://orcid.org/0000-0002-6413-1363

\section{- Acknowledgements}

This work was also supported by the Soonchunhyang University Research Fund. 
2020 Statistics and Best Reviewers | Chung M, et al.

\section{References}

1. Chung M, Kim BT : Editorial statistics and best reviewers award 2018 for Journal of Korean Neurosurgical Society. J Korean Neurosurg Soc 62 :
$1-2,2019$

2. Kim BT, Chung $M$ : Evolution of quality improvement for the Journal of Korean Neurosurgical Society. J Korean Neurosurg Soc 61 : 425426, 2018 